\title{
Practical Considerations for the Development of a Robust Two-Step Dissolution Test for Enteric-Coated Immediate- and Extended-Release Solid Oral Dosage Formulations

\author{
Henry Zhao ${ }^{1, *}$, Stephen Cafiero ${ }^{1}, Z^{2}$ eena Williams ${ }^{1}$, and Kevin C. Bynum ${ }^{2}$ \\ 'Boehringer Ingelheim Pharmaceuticals Inc., Ridgefield, CT \\ 2OSI Pharmaceuticals, Cedar Knolls, NJ
}

\begin{abstract}
The following describes the practical details for developing a robust two-step dissolution test for enteric-coated, immediate- and extended-release solid oral dosage forms with fast HPLC analysis. Two-step dissolution methods for poorly soluble compounds require special considerations, two of which are the addition of surfactant to one (or both) of the dissolution media to achieve sink conditions and the choice of technique utilized to switch medium pH during the test. Robust and repeatable performance should be expected if the proper tools and techniques are utilized. In addition, two-step dissolution methods should provide information about the coating quality as well as the potential for coating failure in the acid step. Finally, the surfactant concentration present in either the acid or buffer stage needs to be optimized and justified.
\end{abstract}

\section{INTRODUCTION}

nteric coating of a tablet or capsule is a technique

- commonly employed to protect a solid oral dosage

form from the acidic environment of the human

stomach. However, the coating will break down rapidly in a neutral environment (pH 5.5 or greater). Enteric-coated tablets and capsules are considered delayed-release formulations because drug release is retarded until the drug product is exposed to the neutral environment of the upper intestinal tract (1). Many solid oral dosage forms require enteric coating because there is a need to sequester the active pharmaceutical ingredient (API) from interaction with the low pH environment of the stomach. This need may arise if the API degrades upon exposure to an acidic environment or causes an undesirable effect (irritation) on the gastric lining (1). Finally, excipients in the dosage form may respond or perform in an unintended way if exposed to an acidic environment.

As stated in USP $\langle 711\rangle$, a two-step dissolution method is needed to determine the integrity of the enteric coating in an acidic environment and to measure the release of the dosage form in a neutral environment (2). For immediate-release solid oral dosage forms that are enteric-coated, USP $<711>$ requires a two-step dissolution method that demonstrates coating integrity in an acidic environment for two hours, followed by exposure to a neutral $\mathrm{pH}$ environment for about one hour $(2,3)$. The test begins with the dosage form in an acidic environment (USP $<711>$ requires $0.1 \mathrm{~N} \mathrm{HCl}$ ), followed by a $\mathrm{pH}$ shift to a

*Corresponding author. neutral environment. The release is measured in a medium with a phosphate buffer at $\mathrm{pH}$ 6-7.

In addition to delayed-release formulations, a two-step dissolution method is also needed to determine the integrity of enteric-coated, extended-release solid oral dosage formulations. In this case, the coating integrity is demonstrated in an acidic environment for two hours followed by release in a neutral $\mathrm{pH}$ environment, typically for up to $24 \mathrm{~h}$. Since enteric-coated, extended-release solid oral dosage formulations target the lower Gl tract, it is important that the release be observed during the buffer stage.

\section{Method Development-Drug Substance Solubility}

Development of a robust dissolution method that will provide relevant information regarding the quality of the enteric coating and the dissolution properties of the solid oral dosage unit requires several considerations. The first concerns the solubility of the drug substance itself. It has become a common practice to utilize synthetic surfactants to aid in the dissolution of poorly soluble compounds $(3,4)$. Surfactant may be added to the dissolution medium to bring the solubility of the drug substance to sink conditions, which is defined as at least three times the thermodynamic solubility limit of the compound under study $(4,5)$. This same consideration should be applied to a two-step dissolution test. The drug substance may have a pH-dependent solubility, so an evaluation of the solubility of the compound in both the acidic and neutral media must be made. The drug substance should be soluble up to the specification level in the acid-step medium to be 
able to detect a failure in the enteric coating. If the specification level for the acidic step is not more than $10 \%$ released, the acid-step medium must be able to dissolve at least $10 \%$ of the active ingredient in the drug product. If this is not the case, an enteric coating failure may occur and not be properly quantitated. If the drug substance is not soluble in the acid-step medium, surfactant may be added to solubilize at least $10 \%$ of the API in the dosage form. The analyst should investigate the type and concentration of surfactant needed to ensure that it does not adversely interact with components of the coating to cause a failure that is not biorelevant. The second-step medium may also contain an appropriate and justifiable amount of surfactant. Theoretically, this will allow the drug substance to be completely solubilized in the final dissolution medium.

\section{Method Development-Medium Addition or Replacement}

The second key consideration in developing a two-step dissolution method is to develop a test that can be easily executed on a routine basis and repeated in a robust manner. Unlike single-step methods, two-step dissolution methods require that the $\mathrm{pH}$ of the dissolution medium be changed during the test. Generally, there are two ways to accomplish this $\mathrm{pH}$ shift during the dissolution test, medium addition or complete medium replacement.

The medium-addition approach begins with exposure of the coated dosage unit to an acidic environment, typically $0.1 \mathrm{~N} \mathrm{HCl}$, for two hours at $37^{\circ} \mathrm{C}$. After two hours, the medium is sampled, and the aliquot is analyzed for any drug that has been released into this acidic environment. If less than $10 \%$ of the drug substance is released from the drug product during this first step, the coating is considered to have "passed" the acid-step test (2).Immediately following the sampling of the acid aliquot, the dissolution vessel contents are adjusted to a neutral $\mathrm{pH}$ with the addition of a concentrated buffer solution that has been preheated to $37^{\circ} \mathrm{C}$. The coated formulation will be exposed to this neutral environment, typically $900-1000 \mathrm{~mL}$ of total vessel volume, for an additional period of time where the enteric coating will break down, and the tablet dissolution can proceed. The buffer concentration in the second-step medium should be at a level that will ensure an accurate shift in $\mathrm{pH}$ upon addition to the acid-step medium. The medium-addition approach requires the addition of a relatively small amount of medium to each vessel in a short period of time (approximately $1-5 \mathrm{~min}$ ). Furthermore, an accurate volume of medium must be added to ensure that a volumetric error does not occur.

The complete medium replacement approach is similar to the medium-addition technique in that the tablet is first exposed to an acid medium for two hours. After two hours, a sample is taken, and the dosage unit is removed from the acidic environment. At this point, the acidic medium may be discarded. The dosage unit is then submerged in a dissolution vessel containing neutral buffer, and the dissolution test is continued in this new medium. In some automated systems, the medium is emptied and refilled without removing the dosage unit from the vessel. In nonautomated systems, the dosage form may be manually moved from one vessel to another (either in a basket that is raised from the bath or, if a paddle apparatus is used, removed from the bottom of the vessel). Using either approach, the neutral-step medium must be preheated, and the change must be made within $5 \mathrm{~min}$. For both methods, the $\mathrm{pH}$ shift must be accomplished in a controlled and reproducible manner.

Our laboratory has focused on the medium-addition technique because it is less invasive for the dosage form (dosage form remains in the vessel) and is easier to conduct in a short time when running multiple baths. We have found that this approach is less labor-intensive and allows for higher sample throughput. This publication will discuss our strategy for developing a robust, two-step dissolution test for an enteric-coated tablet containing a poorly soluble drug substance. The development of dissolution test procedures for enteric-coated, immediaterelease and enteric-coated, extended-release tablets will be reported.

Finally, consideration of the importance of API acid-step solubility and an ethanol dose-dumping study are discussed. The enteric-coated formulation should be designed to be rugged so that dose dumping does not occur in the presence of alcohol. Ethanol dose-dumping experiments are performed in both acid and buffer stages during early product development to mitigate the risks associated with potential dose dumping.

\section{EXPERIMENTAL \\ Samples}

A two-step dissolution method was developed for an enteric-coated, immediate-release 400-mg tablet and three different enteric-coated, extended-release 400-mg tablets, each formulated to obtain different release profiles. The API is a BCS Class II compound.

\section{Surfactant Study}

The first step in our development strategy for this compound was to determine the solubility of the drug substance in media with and without surfactant. The traditional shake-flask procedure was employed, where an excess of drug substance was exposed to a solution for a set period of time ( $2 \mathrm{~h}-$ a relevant time for dissolution testing) and was agitated by shaking (5). At the end of this time, the undissolved material was separated by centrifugation, and the supernatant was assayed. For this study, $0.1 \mathrm{~N} \mathrm{HCl}$ and $50 \mathrm{mM} \mathrm{pH} 6.8$ phosphate buffer were evaluated as controls. In parallel, we performed the same shake-flask test with the above media containing escalating amounts of Tween 80 (a nonionic surfactant) up to $2 \%$. This study allowed us to observe the amount of 
surfactant that is needed to solvate the drug substance within a time that is relevant to our dissolution test.

\section{Dissolution Method}

All dissolution tests were conducted in a calibrated USP compliant dissolution bath with paddles (USP Apparatus 2) set to rotate at $50 \mathrm{rpm}$. The two-step test was conducted with the medium-addition technique. The initial dissolution step was conducted for $2 \mathrm{~h}$ in $700 \mathrm{~mL}$ of $0.1 \mathrm{~N}$ $\mathrm{HCl}$ containing $0.64 \%$ Tween 80 . Tween 80 was needed to solubilize the drug substance, which is nearly insoluble in both the acidic- and neutral-step media. After two hours, the contents of each dissolution vessel were sampled, and the amount of drug released was assayed. An automated syringe-based autosampler, equipped with $10-\mu \mathrm{m}$ full-flow filters, was utilized to pull samples from the vessels.

After acid-step samples were drawn for measurement, $200 \mathrm{~mL}$ of buffer was accurately added to each vessel. The buffer contained an exact amount of sodium hydroxide needed to neutralize the acid present in the first step as well as a concentrated phosphate buffer. The buffer volume addition was controlled by a custom-made "to dispense" (TD) volumetric flask. Six flasks were utilized (one for each tablet tested). Each was placed in a water bath set to $37^{\circ} \mathrm{C}$ before the medium switch time. Each volumetric flask was filled at room temperature because the flasks are calibrated at $20^{\circ} \mathrm{C}$. The concentrated buffer was subsequently diluted with the first-step medium to contain $0.5 \%$ Tween 80 in 50 mM sodium phosphate as the final medium. Sampling was conducted using a syringe-based autosampler, and samples were analyzed to generate a multipoint dissolution profile.

\section{HPLC Assay}

All samples were assayed by HPLC with UV detection. The HPLC data collection time was 3 min per sample injection. The HPLC conditions listed below are an example of a fast HPLC method that consumes approximately $1.5 \mathrm{~mL}$ of mobile phase per injection, which greatly minimizes the volume of solvent waste generated. HPLC conditions:

$\begin{array}{ll}\text { Column: } & \text { Waters Xbridge C18, 2.5 } \mu \mathrm{m}, \\ & 2.1 \times 30 \mathrm{~mm} \\ \text { Mobile Phase: } & 50 \mathrm{mM} \mathrm{pH} 4.5 \text { acetate buffer/ } \\ & \text { acetonitrile (55/45) } \\ \text { Flow Rate: } & 0.5 \mathrm{~mL} / \mathrm{min} \\ \text { Injection Volume: } & 5 \mu \mathrm{L} \\ \text { Column Temperature: } & 40^{\circ} \mathrm{C} \\ \text { Detection: } & \mathrm{UV} \text { at } 360 \mathrm{~nm}\end{array}$

\section{Dissolution Test Evaluation}

Several enteric-coated drug product formulations were required for the clinical development of this product. An enteric-coated, immediate-release tablet and a series of three enteric-coated, extended-release tablets were formulated. An appropriate dissolution method was needed to test these formulations. The above dissolution method was utilized to measure the quality of the drug product and to serve as a stability-indicating tool. The combined two-step medium in the final test method contained enough surfactant (Tween 80 ) to ensure sink conditions, or at least three times the solubility limit, at two hours. The surfactant concentration was determined based on the data from the surfactant study.

\section{RESULTS AND DISCUSSION Buffer Addition}

Manual medium addition to shift the $\mathrm{pH}$ accurately from low to neutral for a two-step dissolution method can be challenging and labor intensive. We were able to complete this $\mathrm{pH}$ shift accurately using stoichiometry. The concentrated buffer (second-step) medium must contain enough base to neutralize the acid in the first-step medium and must contain a proper buffer ratio to maintain the desired $\mathrm{pH}$ when added. An accurate control of mass and volume is desirable. The concentrated buffer should be carefully prepared and accurately delivered. Customized Class A TD volumetric glassware is recommended for timely and accurate addition of the $200 \mathrm{~mL}$ of buffer-step medium. The $200-\mathrm{mL}$ volumetric flask can be filled at ambient and heated in a water bath to $37^{\circ} \mathrm{C}$ before the buffer step addition (Figure 1). The $\mathrm{pH}$ of the combined media can typically be within $\pm 0.1 \mathrm{pH}$ units of the desired $\mathrm{pH}$ if great care is taken during media preparation and delivery. If combined medium $\mathrm{pH}$ is a critical factor in the measurement of product release, the analytical method should be appropriately validated for robustness to determine the acceptable $\mathrm{pH}$ tolerance.

\section{Surfactants}

For BCS Class II compounds, the use of an appropriate amount of surfactant must be justified for the acid or buffer medium. Sink conditions should be determined for

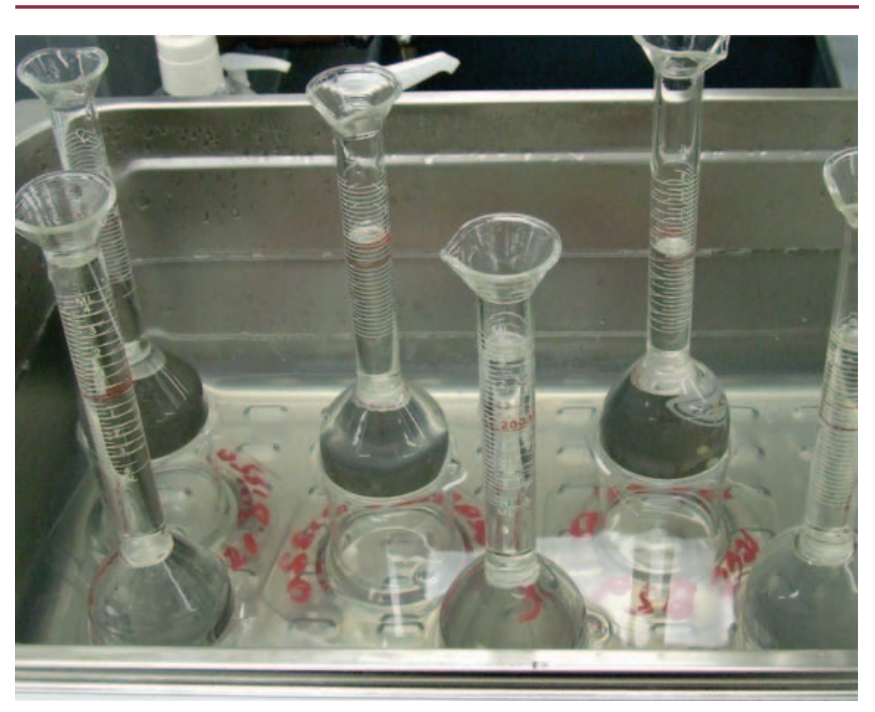

Figure 1. Preheated buffer stage media in 200-mL TD volumetric flask. 




Figure 2. Solubility curve of API in $0.1 \mathrm{~N} \mathrm{HCl} \mathrm{vs.} \mathrm{surfactant} \mathrm{concentration.}$

the use of surfactants, specifically for the combined medium. To determine sink conditions, we have used the shake-flask approach. Saturated solutions containing the API in $0.1 \mathrm{~N} \mathrm{HCl}$ and $\mathrm{pH} 6.8$ phosphate buffers with escalating amounts of surfactant were shaken until thermodynamic equilibrium was reached. Each saturated solution was centrifuged, and the resulting supernatant was analyzed. Saturated solutions prepared in the same manner in the same media without surfactants were used as controls. The results were plotted, and a linear distribution of the solubility curve was achieved (Figures 2 and 3). From the solubility curve, the appropriate surfactant concentration to achieve sink conditions was calculated. If the dosage strength changes, the surfactant concentration to achieve sink conditions can easily be recalculated from the same plot, assuming the sample concentration is within the same linear range. For the tested compound, the media chosen were $700 \mathrm{~mL}$ of $0.1 \mathrm{~N}$ $\mathrm{HCl}$ containing $0.64 \%$ Tween 80 for the first step and $200 \mathrm{~mL}$ of concentrated buffer without surfactant for the second step. The combined medium contained 0.5\% Tween 80 in $\mathrm{pH} 6.8$ phosphate buffer to give sink conditions for the API. The type of surfactant used in two-step dissolution medium development should take into account compatibility with the chosen medium $\mathrm{pH}$, as well as any unintentional dissolving of the enteric coating in the acid step that is not $\mathrm{pH}$-related.

In parallel, the solubility of an existing commercial drug product, sulfasalazine delayed-release tablets, was evaluated to determine its solubility in $900 \mathrm{~mL}$ of $0.1 \mathrm{~N}$ $\mathrm{HCl}$ at $37^{\circ} \mathrm{C}$. This test was executed according to the acid-step test in the USP monograph for sulfasalazine delayed-release tablets (6). In our test, $500 \mathrm{mg}$ of sulfasalazine (Fluka) was spiked into baskets (USP Apparatus 1 at $100 \mathrm{rpm}$ ) for the 2-h duration of the acid step. This test was conducted without placebo. After two hours, a sample was manually pulled and centrifuged at $4000 \mathrm{rpm}$ for $10 \mathrm{~min}$, and the supernatant was analyzed. UV analysis was performed at $356 \mathrm{~nm}$ using standards

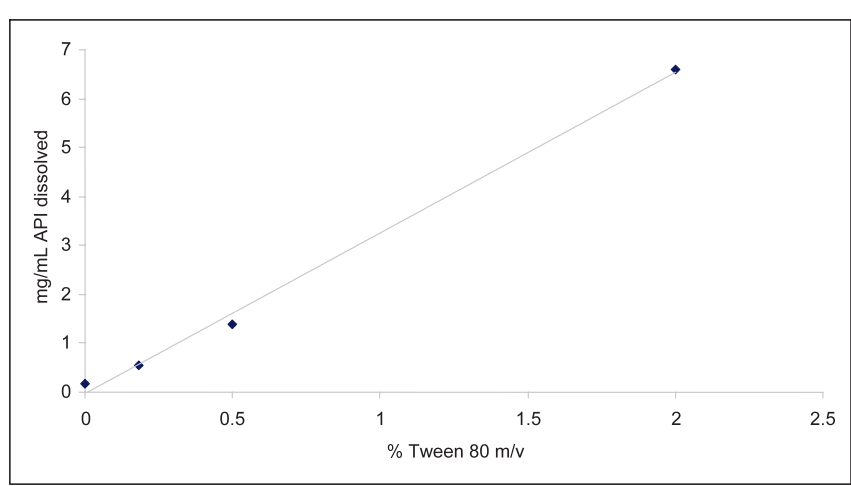

Figure 3. Solubility curve of API in pH 6.8 vs. surfactant concentration.

prepared in 1/1 methanol/0.1 $\mathrm{N} \mathrm{HCl}(\mathrm{v} / \mathrm{v})$. The results showed that no dissolution (or recovery) was observed for sulfasalazine using the USP monograph for the acid step without the use of placebo. While these results may have been different if the test was conducted in the presence of placebo, the crucial point is that it is difficult to fully assess the integrity of the enteric coating without appropriately validating the analytical method to recover at least $10 \%$ of the label claim in the acid-step medium to safely meet the USP specification.

\section{Ethanol Dose-Dumping Experiment}

During method development, the effect of ethanol on the enteric coating of delayed-release formulations should be evaluated. It is critical to examine the coating integrity when these formulations are exposed to $40 \%$ ethanol in the total volume of dissolution medium, which is a worst-case scenario. For modified-release formulations, exposure in up to $40 \%$ ethanol must not induce dose dumping (7). Negative ethanolic effect on the enteric coating and formulation release can have a significant impact on the drug product efficacy and may require special labeling. The test should be performed during early formulation development to minimize these risks.

The ethanolic effect experiment for our BCS Class II compound was performed by volumetrically replacing medium with ethanol. The acid-step medium was prepared by mixing approximately $8 \mathrm{~mL}$ of ACS grade $\mathrm{HCl}$ with each liter of $40 / 60$ ethanol/water ( $/ \mathrm{v})$.

Using the above approach, a discriminating dissolution method was developed (Figures 4 and 5). Additionally, the developed method was stability-indicating (Figure 6).

\section{CONCLUSION}

A two-step dissolution method is needed to evaluate the quality of an enteric-coated formulation. A surfactant study may be employed to optimize the surfactant concentration to achieve sink conditions. The stoichiometric addition of buffer can help to accurately shift medium $\mathrm{pH}$ if careful technique is utilized. Even with careful technique and practice, this is a labor-intensive process 




Figure 4. Dissolution profiles for immediate-release core tablets and enteric-coated tablets.

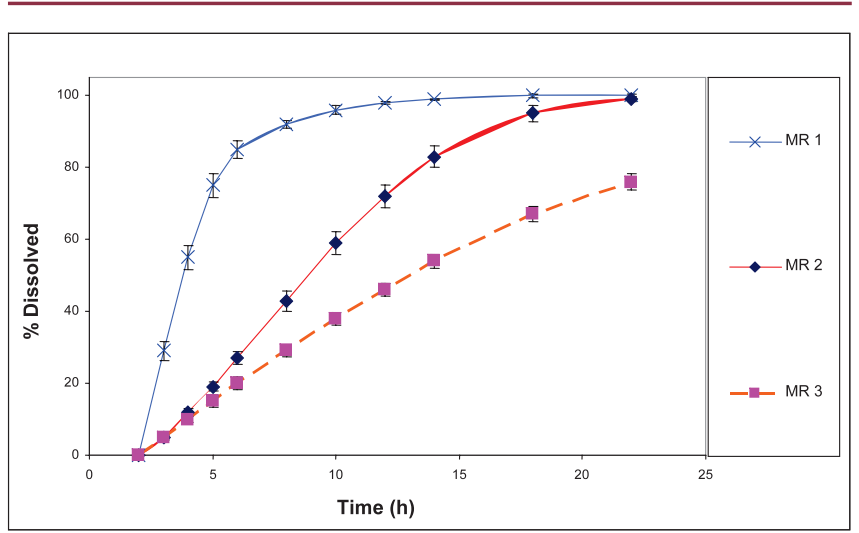

Figure 5. Dissolution profiles for modified-release formulations. Method discriminates three different formulations.

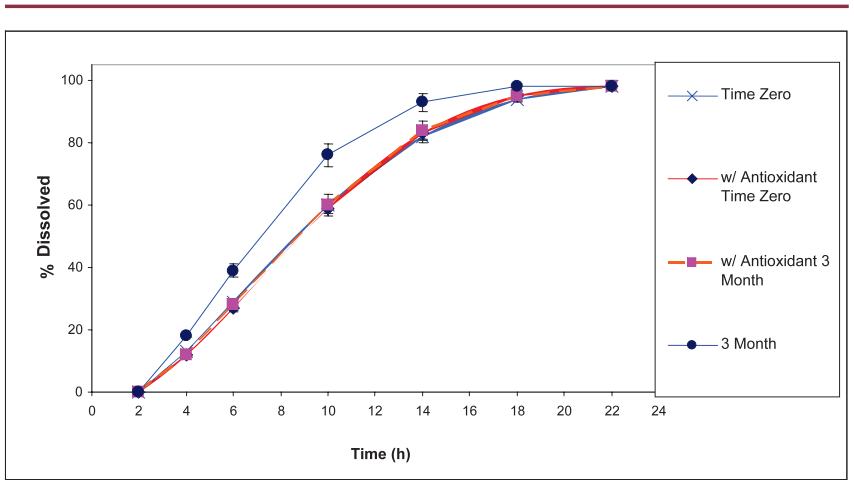

Figure 6. Stability-indicating method for modified-release formulation change in release profile at 3 months without antioxidant in the formulation. with an opportunity for future automation. Finally, studies may be needed to demonstrate that ethanol will not induce dose dumping in enteric-coated formulations in both acid and buffer steps. By using this approach, an accurate and stability-indicating dissolution method for both immediate-release and modified-release enteric-coated formulations can be developed.

\section{REFERENCES}

1. Bruce, D. L.; Petereit, H.-U.; Beckert, T.; McGinity, J.W. Properties of enteric-coated sodium valproate pellets. Int. J. Pharm. 2003, 264, 85-96.

2. $\quad<711>$ Dissolution. In United States Pharmacopeia and National Formulary USP 32-NF 27; The United States Pharmacopeial Convention, Inc.: Rockville, MD, 2009.

3. <1092> The Dissolution Procedure: Development and Validation. In United States Pharmacopeia and National Formulary USP 32-NF 27; The United States Pharmacopeial Convention, Inc.: Rockville, MD, 2009.

4. Jamzad, S.; Fassihi, R. Role of Surfactant and pH on Dissolution Properties of Fenofibrate and Glipizide-A Technical Note. AAPS PharmSciTech 2006, 7 (2), Article 33.

5. Gupta, A.; Gaud, R. S.; Ganga, S. Development of Discriminating Dissolution Method for an Insoluble Drug: Nisoldipine. Int. J.Pharm. Tech. Res. 2010, 2 (1), 931-939.

6. Sulfasalazine Delayed-Release Tablets. In United States Pharmacopeia and National Formulary USP32-NF27; The United States Pharmacopeial Convention, Inc.: Rockville, MD, 2009.

7. Meyer, R. J.; Hussain, A. S. Awareness Topic: Mitigating the Risks of Ethanol Induced Dose Dumping from Oral Sustained/Controlled Release Dosage Forms. FDA's Advisory Committee for Pharmaceutical Science Meeting, Rockville, MD, October 25-26, 2005. http:// www.fda.gov/ohrms/dockets/ac/05/briefing/ 2005-4187B1_01_08-Alcohol-Induced.pdf (accessed Jan 20, 2011). 